PIOTR GotOWKO, Zürich

\title{
Die interne Rechtssetzung und die Rechtsdurchsetzung im Deutschen Orden
}

The legislation in the Teutonic Order started just after its founding in 1191, at latest after his rising to the rank of a military order in 1198. In the first phase the brethren simply took over the rule of the Templars with some supplements from the rule of the Hospitallers. With the development of their own legal ideas around 1210-1215 the second legislative phase began. At its end the Teutonic Knights revised their legislative acts entirely and replaced them with new, written in a book called "Ordensbuch" - the Book of the Order - and consisted of Rules, Laws and Customs. In every castle of the Teutonic Order a copy of such a Book should be present and read to all the members once a week to allow them a thorough legal knowledge. In few cases the legal acts proved insufficient or not clear enough so the later Grandmasters developed or changed them. So began the third and last step in the legislation. In each of those three phases the general chapter - an assembly of all the brethren of the Order-played a crucial role for without their permission no legal act could be launched. A strict system of punishments applied by the local chapters made sure that the regulations of the Brotherhood were observed thus providing a main function in the law enforcement.

\section{Die Rechtssetzung in der Anfangszeit}

Die Rechtssetzung dürfte im Deutschen Orden gleich nach seiner Gründung im Jahre 1191, spätestens jedoch nach seiner Erhebung zum geistlichen Ritterorden 1198 begonnen haben, wobei die Vorgänge sich in drei unterschiedliche Phasen einteilen lassen. Die Bruderschaft benötigte gut funktionierende Strukturen in ihrem Inneren, zu denen ein für die Mitglieder verbindliches Recht gehörte, um die Hauptaufgaben - den Schutz der Christen und die Bekämpfung von Ungläubigen - überhaupt erfüllen zu können. So sollten die Brüder nach der Umformung in den Ritterorden einstimmig beschlossen haben, in allen Belangen die Templerregel zu befolgen, außer bei der Krankenpflege, wofür die Regel der Johanniter gelten sollte. ${ }^{1}$ Nach der Bestätigung des neuen Ritterordens durch den Papst Innozenz III. am 19. Februar 1199 wurde vom Heiligen Stuhl wiederholt, dass die Deutsche Bruderschaft sich bei allen Belangen nach der Regel der Templer, beim Spitalwesen nach derjenigen der Johanniter leben sollte. ${ }^{2}$ Diese erste Phase der Rechtssetzung legte den Grundstein in Bezug auf die inneren Strukturen und das Innenleben, stellte jedoch zugleich nichts anderes als eine Übernahme der Rechtsnormen von den bestehenden zwei Ritterorden

\footnotetext{
1 Perlbach, Statuten 160: „Narracio“, Punkt 4; Perlbach edierte im genannten Werk die Ordensbestimmungen, die bis heute als eine herausragende Sammlung des Quellenmaterials gelten.

2 STREHLKE, Tabulae Ordinis Theutonici Nr. 297; SARNOWSKY, Der Deutsche Orden 15f.; BuSCHINGER, OLIVIER, Les chevaliers teutoniques 31ff.; BOOCKMANN, Der Deutsche Orden 32 ff.
} 
dar, mit denen sich die Deutschen Brüder ununmittelbar danach schrittweise vertraut machten.

\section{Die zweite Phase der Rechtssetzung}

Mit der Entwicklung der eigenen RechtsvorstelRechtsvorstellungen ab zirka 1210-1215 wird die zweite Legislativphase eingeläutet. Die Brüder ergänzten ihre Normen durch alltägliche Bräuche, änderten sie ab und entwickelten neue. Urkunden zu diesen Vorgängen lassen sich nicht finden, wohl deshalb, weil sie mündlich geschahen. Als mit den Templern ein Streit um die weissen Mäntel entflammte, deren Farbe der mächtige Templerorden ausschliesslich für sich beanspruchte, entschied Papst Honorius III. im Jahre 1221, dass auch Angehörige der Deutschen Bruderschaft ihre Mäntel in weiß tragen dürfen, wie es ihren Gewohnheiten entspreche. ${ }^{3}$ Folglich muss zu dieser Zeit der Deutsche Orden erste selbstentwickelte Rechtsbräuche gekannt haben. Die rechtliche Verselbständigung nahm mit dem Zeitablauf $\mathrm{zu}$, wobei der Einfluss und die Macht, zu denen die Bruderschaft unter dem damals amtierenden Hochmeister Hermann von Salza gelangte, diese Vorgänge gefördert haben dürfte. Im Frühjahr 1244 baten die Brüder schließlich den Papst um Streichung von fünf nicht mehr beachteten Bestimmungen aus der Ordensregel. ${ }^{4}$ In der Bulle vom 9. Februar 1244 hob Innozenz IV. diese auf und ermächtige das Kapitel außerdem, weitere Bestimmungen zu ändern. ${ }^{5}$ Diese päpstliche Erlaubnis nutzten die Brüder, um zwischen 1244-49 ihre Normen

\footnotetext{
${ }^{3}$ STREHLKE, Tabulae Ordinis Theutonici Nr. 308.

${ }^{4}$ PerlbaCH, Statuten XLVII; Wie Perlbach nachweist, stimmen alle fünf Bestimmungen mit der Templerregel überein.

${ }^{5}$ StREHLKE, Tabulae Ordinis Theutonici Nr. 470.
}

gänzlich zu überarbeiten. ${ }^{6}$ So gipfelte die zweite Phase der Rechtssetzung in der Revision der für die Bruderschaft verbindlichen Rechtsnormen. An deren Ende schufen sich die Brüder ein eigenes internes Recht, bestehend aus drei Teilen, die sie selber als Regeln ("regele“), als Gesetze ("gesetzede") und als Gewohnheiten (,"gewonheite") bezeichneten.7

\section{Zur Herkunft der Begriffe}

Der erste Teil, die Regeln, dürfte so in der Anlehnung an die Bestimmungen der Templer geheißen haben, die ihre Normen "La Règle" nannten. ${ }^{8}$

Die Begriffsherkunft des zweiten Teils, der Gesetze, lässt sich hingegen nicht eindeutig ermitteln. Sterns sucht nach einer möglichen Ursache für die Entstehung der Gesetze in den ersten Jahrzehnten der Ordensgeschichte. So wurden auf dem Vierten Laterankonzil, welches von Papst Innozenz III. im Jahre 1215 einberufen wurde, um die Kirche zu reformieren und einen neuen Kreuzzug vorzubereiten, auch einige Beschlüsse gefasst, die die Normen des Deutschen Ordens beeinflussten. Auf jenem Konzil wurde unter anderem den Mönchen verboten, Beschwerden gegen die Entscheidungen ihrer Äbte einzulegen. Ein ähnliches Verbot findet sich im Gesetz II.d des Deutschen Ordens, in dem solche Brüder, die "wider ihres ordenes gesezzede sich anderswâr berûfent oder appelîrent", mit Jahresbuße belegt werden. Im Abschnitt "De vita et honestate clericorum" wird den Mönchen Trunkenheit und häufiges Aufsuchen von Gasthöfen untersagt, was den Brüdern des Deutschen Ordens

\footnotetext{
${ }^{6}$ Perlbach, Statuten XLVIII; Militzer, Von Akkon zur Marienburg 49f.; Sterns, Statutes 46f.; BISKUP, LABUDA, Dzieje Zakonu Krzyżackiego w Prusach 166f. 7 PERLBACH, Statuten 29, 57, 90.

${ }^{8}$ UPTON-WARD, Rule of the Templars 2 ff.; DE CURZON, Règle du temple 2 ff.; DEMURGER, Ritter des Herrn 92.
} 
ebenfalls nicht gestattet war. Mönche, die unterwegs waren, sollten gemäß den Beschlüssen des Laterankonzils Orte mit schlechtem Ruf meiden und den Würfelspielen fernbleiben. Das Gesetz III weist gewisse Schnittstellen auf, denn es klassifiziert Spiele und Trinken außerhalb des Ordenshauses als leichtere Vergehen. Die Einflüsse des Vierten Laterankonzils kann man ferner auch bei den Regeln und den Gewohnheiten erkennen. Das Konzil hielt fest, dass die Almosensammler Mässigung in allen Lebensbereichen zeigen sollten und warnte insbesondere vor Unterkünften in Schankwirtschaften, verschwenderischem Ausgeben oder unangebrachter Kleidung. Das Kap. 7 der Deutschordensregel verlangte von den "almûsen bittere" gute Lebensführung und Zurückhaltung in den Ausgaben. In einem anderen Dekret wurde der Ablauf der Wahlen geregelt. Wählen sollten entweder alle Brüder oder eine gewisse Anzahl von ihnen, die ihr ganzes Konklave repräsentieren müssen. ${ }^{9}$ Die Deutschen Brüder befolgten strikt diese Anweisung und entschieden sich für die zweite Alternative, die in Kap. 5 der Gewohnheiten zum Ausdruck kommt: Die Hochmeisterwahl sollte in einem Wahlgremium mit 13 Anwesenden stattfinden und entweder einstimmig oder mehrheitlich ergehen. ${ }^{10}$ Somit dürften die Dekrete des Vierten Laterankonzils einige Bestimmungen im Deutschen Orden beeinflusst haben, der die innenkirchliche Diskussion mitverfolgte. ${ }^{11}$ Es ist möglich, dass die Brüder für solche vom Vierten Laterankonzil beeinflussten Beschlüsse einen neuen Oberbegriff erfanden. Gegen eine solche These spricht jedoch, dass die Einflüsse des Laterankonzils nicht nur in Gesetzen, sondern auch in den Regeln und den Gewohnheiten erkennbar sind und zudem sehr allgemeine Ausrichtung haben, die der Deutsche Orden unabhängig von innen-

\footnotetext{
${ }^{9}$ STERNS, Statutes $99 \mathrm{ff}$.

${ }^{10}$ PerLbach, Statuten 93ff., Gewohnheiten 4-7.

${ }^{11}$ STERNS, Statutes $104 \mathrm{f}$.
}

kirchlichen Reformversuchen hätte erlassen können.

Eine andere Ursache für die Entstehung der Gesetze könnten äußere Rechtssetzungsakte in Form von päpstlichen Beschlüssen gewesen sein. Dagegen sprechen Kap. II und Kap. 31 der Gesetze, die auf die innere Beschlussfassung im Orden hinweisen. Wie bereits erwähnt, erlaubte der Papst im Jahr 1244 dem Orden, seine Bestimmungen selber zu ändern. Daraus lässt sich schlussfolgern, dass die Gesetze kaum vom Papst aufgezwungen worden sein können. Ebenso wenig wahrscheinlich ist es, dass den Gesetzen Rechtssetzungsakte des Kaisers zugrunde liegen. Zwar beobachtet man im Abendland zwischen 1100-1250 eine enorme Zunahme von juristischen Aktivitäten und ab 1250 ein Ergreifen der Legislativbefugnisse durch die Obrigkeitsträger, ${ }^{12}$ die Deutschen Brüder unterstanden als geistlicher Ritterorden jedoch keinen weltlichen Herrschern, sondern direkt dem Heiligen Stuhl.

Die Gesetze dürften auch als ein Ergebnis der autonomen Beschlussfassung des Ordens angesehen werden. Der Aufbau scheint, dies zu bestätigen, denn zu Beginn werden die Gesetze mit römischen Ziffern als Gesetz I, II und III bezeichnet, als viertes Gesetz folgt aber Gesetz 1, gefolgt von Gesetz 2 usw., woran man ihre schrittweise Entstehung erkennt. Die wohl älteste Norm in diesem Teil, das Gesetz I, listet Bestimmungen auf, die in der Temperregel nicht wiederzufinden sind, wie etwa Verbote, sich barfuss zur Kirche zu begeben, Alkohol zu konsumieren oder Märkte zu aufzusuchen. ${ }^{13}$ Der Inhalt von Gesetz II stützt die vorab erwähnte Theorie, da darin die Einberufung des Ordenskapitels erörtert wird, welches als oberstes Rechtssetzungsorgan im Orden tätig war. Auch die späteren Hochmeister nannten ihre Rechts-

\footnotetext{
${ }^{12}$ KRAUSE, Gesetzgebung 1606-1620.

13 STERNS, Statutes 73.
} 
setzungsakte "gesetzede“. Dieser Begriff dürfte diejenigen Beschlüsse umschreiben, die autonom innerhalb der Bruderschaft entstanden, also "gesetzt" worden sind.

Die Gewohnheiten stellten den dritten Rechtsteil dar. Ihre Entstehung wirft, ähnlich wie die der Regeln, deutlich weniger Fragen auf. So bezeichneten die Brüder ihre im Laufe der Zeit entwickelten Bräuche. Diese drei Teile - die Regeln, die Gesetze und die Gewohnheiten wurden in einem Buch zusammengefasst, welches man das Ordensbuch („ordenbûch“) nannte. Eine Abschrift hiervor sollte auf jeder Burg vorhanden sein, auf der die Brüder lebten. Die zahlreichen bis heute erhaltenen Ordensbücher belegen, dass dieser Forderung gänzlich entsprochen wurde. ${ }^{14}$

\section{Das letzte Legislativstadium}

$\mathrm{Ab}$ der zweiten Hälfe des 13. Jahrhunderts begann schließlich der dritte Abschnitt in Rechtssetzung, der dem vorangehenden stark ähnelte, denn auch er zeichnete sich durch Ergänzung und Entfaltung der bestehenden Bestimmungen aus. Anders als bei der zweiten Phase kam es jedoch zu keiner umfassenden Revision mehr, was auch beabsichtigt war, damit die bereits bestehenden, aufwendig kopierten Ordensbücher weiterhin in Kraft bleiben konnten. Die neuen Rechtsakte wurden lediglich am Ende jener Ordensbücher angefügt. ${ }^{15}$ Insbesondere das 14. Jahrhundert brachte viele solcher Normen hervor, die die Brüder "gesezzede" oder "gesetze" nannten und die deshalb als Gesetze späterer Hochmeister bekannt wurden. ${ }^{16}$ Sie

\footnotetext{
14 POTKOWSKI, Duchowość Krzyżaków a lektura 94; MentZel-Reuters, Arma spiritualia 105ff; Perlbach, Statuten, X-XXIX..

${ }^{15}$ MiLITZER, Von Akkon zur Marienburg 49f.; PERLBACH, Statuten XLVII; STREHLKE, Tabulae Ordinis Theutonici, Nr. 100; STERNS, Statutes $49 f$.

16 Perlbach, Statuten 134, 148.
}

bildeten nun den vierten und zugleich letzten Teil der Ordensbücher.

\section{Die Rolle des Ordenskapitels}

Bei jeder dieser drei Phasen, insbesondere seit Erlangung der rechtssetzenden Unabhängigkeit in der dritten Phase, spielte das große Ordenskapitel eine gewichtige Rolle. Darunter ist die Versammlung der wichtigsten Amtsträger im Orden zu verstehen, des Hochmeisters, der Großgebietiger, des Deutsch- und des Livländischen Meisters und der Komture. Es fand einmal jährlich am 14. September nach der Kreuzerhöhung statt. ${ }^{17}$ Neben der Legislativtätigkeit behandelte das große Kapitel weitere wichtige Probleme: die geistliche und die materielle Lage des Ordens, den Stand seiner Werke und seiner internationalen Beziehungen. Über die genauen Abläufe ist wenig bekannt, da alles geheim gehalten wurde. Als sicher gilt, dass es mit einer feierlichen Messe begann und jeder, der den Saal betrat, sieben Mal das Vaterunser beten musste. Die Gebete sollten nicht nur göttlichen Beistand ersinnen, sondern auch die Brüder erneut auf die Wichtigkeit ihrer Aufgabe aufmerksam machen. Der Hochmeister öffnete und leitete die Beratungen, die versammelten Gebietiger und die übrigen Brüder saßen rings umher. ${ }^{18}$ Kein Bruder durfte sich während der Beratungen fremdartigen Themen widmen. Wo Handlungsbedarf bestand, wurde über neue Gesetze sowie die Veränderung oder die Vervollständigung der bestehenden Gesetze beraten. Als Organ übte das große Kapitel die Gesetzgebung für den ganzen Orden aus. Der Hochmeister durfte zwar die Gesetze mit Beirat der Großgebietiger entwerfen, sie galten aber

\footnotetext{
17 PerlbaCH, Statuten 102, Gewohnheit 18; JÓźWIAK, Centralne organy władzy 129; BISKUP, LABUDA, Dzieje Zakonu Krzyżackiego w Prusach 274.

18 VoIGT, Geschichte Preußens VI, 435.
} 
erst, wenn das große Kapitel sie bestätigt hatte. Auch konnten bereits bestehende Normen nur durch den Beschluss des großen Kapitels aufgehoben oder verändert werden. Dem Hochmeister allein stand diese Befugnis nicht zu. Auch er war als wichtigster Amtsträger im Orden dem großen Kapitel in seinem Verhalten verantwortlich. Wollten die Brüder rechtliche Inhalte verabschieden oder abändern, so wurde darüber abgestimmt. Entschieden wurde nach der Mehrheit der Stimmen, wobei jeder ein Stimmrecht hatte. Was die erfahrenen Brüder, der sogenannte bessere Teil, rieten, dem sollte man folgen. ${ }^{19}$ Bei geteilten Meinungen dürfte der Hochmeister den Stichentscheid gehabt haben. ${ }^{20}$ Neben der Rechtssetzung kamen dem großen Kapitel noch zwei weitere zentrale Funktionen zu: die Kontrolle und die Repräsentation. Nur im Großen Kapitel wurden die wichtigsten Ordensämter verliehen und schwerwiegendste Vergehen der Ordensbrüder geahndet. ${ }^{21}$ Dort kamen ebenfalls die Brüder aus allen Landesteilen zusammen, wo der Orden seine Niederlassungen hatte. So betrachtet stützten sich die Entscheide des großen Kapitels auf eine sehr breite demokratische Basis. Der Ort, an dem das Kapitel gehalten wurde, war stets an den Sitz des Hochmeisters und des Haupthauses gebunden. Anfänglich fand es in Akkon statt, nach dem Verlust des Heiligen Landes 1291 tagte es einmal jährlich in Venedig, wo die Zentrale vorübergehend residierte. Nach der Übertragung des Hauptsitzes nach Preußen im Jahr 1309 wurden die großen Kapitel auf der Marienburg gehalten. Ein für damalige Verhältnisse herausragendes Korrespondenznetz sorgte dafür, dass

\footnotetext{
19 Perlbach, Statuten 49, Regel 27; VoIGt, Geschichte Preußens VI, 435.

${ }^{20}$ PerlbaCH, Statuten 49, Regel 27.

${ }^{21}$ PerlbaCH, Statuten 102, Gewohnheit 18; 96, Gewohnheit 7a; 59, Gesetz II(a); Andere Ämter wurden an Landkapiteln zugeteilt; PERLBACH, Statuten 59, Gesetz II(b).
}

die Kapitelbeschlüsse rasch an Brüder in allen Landesteilen Preußens, Livlands und in die Kommenden des Reiches befördert wurden.

\section{Mittel zur Rechtsdurchsetzung}

Was die Deutsche Bruderschaft für ihre Angehörige an Recht gesetzt hatte, versuchte sie durch ein breites Instrumentarium an Mitteln durchzusetzen, die im zweiten Teil der Ordensbücher, den Gesetzen, behandelt wurden. Die Brüder der jeweiligen Burgen trafen sich an Sonntagen zu den sogenannten Kapiteln („,capitel“), wo sie unter anderem ihre Vergehen gestehen mussten.22 Verschwieg ein Bruder seine Untat, so hatten andere Ordensangehörige die Pflicht, ihn anzuzeigen, was der Rechtsdurchsetzung sehr förderlich war. Wenn ein Bruder straffällig wurde, kannte der Orden vier unterschiedliche Kategorien in der Bestrafung: die leichte („schult"), die schwere (swêre schult"), die schwerere ("swêrer schult") sowie die schwerste (",aller swêreste schult").23

Die leichte Schuld, wie der Quellenbegriff für die erste von vier Strafstufen lautete, wurde vor allem für die unerlaubte Entfernung aus dem Ordenshaus für kurze Zeit, das längere Ausblieben als es für die Erledigung der Angelegenheiten notwendig, das Reiten ohne Erlaubnis in eine Stadt sowie das gemeinsame Essen oder Trinken mit Weltlichen verhängt. Zur Strafe wurde der Bruder an einem, zwei oder drei Tagen ausgepeitscht. ${ }^{24}$

Das Verbringen einer Nacht außerhalb des Ordenshauses, die Beförderung fremder Briefe ohne Erlaubnis, der grundlose Kontakt zu Welt-

\footnotetext{
22 Perlbach, Statuten 78, Gesetz 34; GòRSKI, Zakon Krzyżacki 87.

${ }^{23}$ Perlbach, Statuten 80ff., Gesetze 36-39.

${ }^{24}$ PerlbaCH, Statuten 80, Gesetz 36 (5); 81, Gesetz 36, 36(6). Die genaue Zumessung der Strafdauer wurde der wöchentlichen Versammlung der Brüder auf dem Kapitel übertragen; GÒRSKI, Studia i materiały $200 f$.
} 
lichen mit schlechtem Ruf, das ausschweifende Essen und Trinken oder Handgreiflichkeiten gegen andere Brüder zogen bereits die zweite Strafstufe, nämlich die schwere Schuld, nach sich. ${ }^{25}$ Als Strafe hierfür war die Jahresbuße vorgesehen. Der Bruder erhielt an jedem Sonntag während eines Jahres Auspeitschung und musste weitere Sanktionen hinnehmen, wie etwa Verlust des Mantels und der Rüstung, karge Speisen aus Wasser und Brot an drei Tagen pro Woche, Essverbot mit anderen Brüdern und am gleichen Tisch oder Zwang, mit Kriegsgefangenen zu arbeiten. ${ }^{26}$

Die schwerere Schuld, wie die Brüder die dritte Strafstufe nannten, verhängte man für das Verbringen von zwei oder mehr Nächten ohne Erlaubnis außerhalb der eigenen Burg, die kurzfristige Entfernung wider ausdrückliche Weisung des Vorgesetzten, Begehen eines Diebstahls, die Haltung von Eigentum, die Sünde mit einer Frau oder das eigenwillige Verlassen des Ordens. ${ }^{27}$ Die Sanktionen waren die gleichen wie bei der Jahresbuße. An Sonntagen wurde der Täter während eines Jahres ausgepeitscht, er verlor für die Strafdauer seine Rüstung und seinen Mantel mit dem Kreuz, aß lediglich Wasser und Brot drei Tage pro Woche, wobei er nicht mit anderen Brüdern speisen durfte. Bei der schwereren Schuld konnte die Strafe um ein weiteres Jahr verlängert werden. Verhielt sich der bestrafte Bruder ungebührlich, so konnten ihn andere Brüder in Eisen legen und bei anhal-

\footnotetext{
${ }^{25}$ Perlbach, Statuten 82, Gesetz 37 (2)-(12); ScHMIDT, Handhabung der Strafgewalt 103ff.; JóźWIAK, TRUPINA, Organizacja życia 46.

${ }^{26}$ PERLBACH, Statuten 82: Gesetz 37; Die Strafdauer wurde in jedem Fall einzeln bemessen, durfte aber ein Jahr nicht überschreiten; GóRSKI, Zakon Krzyżacki $87 \mathrm{ff}$.

${ }^{27}$ Perlbach, Statuten 83f., Gesetz 38 (1)-(10).
}

tender Uneinsichtigkeit sogar eine zeitlich unbegrenzte Einkerkerung verfügen. ${ }^{28}$

Unter die vierte und letzte Strafkategorie, die schwerste Schuld, fiel all das, was man als die schwerwiegendsten internen Verbrechen gegen die Bruderschaft auffasste. Dazu zählten insbesondere der Ämterkauf, die Flucht vor dem Feind, der Übertritt zum heidnischen Glauben, das Verschweigen eines Grundes, der die Aufnahme in die Bruderschaft verunmöglichen würde. Die letztgenannte Tat durfte verziehen werden, in den anderen Fällen wurde der Übeltäter aus dem Ritterorden in einen reinen Mönchsorden mit strengerer Disziplin vertrieben. ${ }^{29}$ Diese Strafen mögen auf den ersten Blick sehr streng, ja grausam erscheinen. Wie Górski in seinen wegweisenden Arbeiten betonte, war die Feudalritterschaft undiszipliniert, der bedingungslose Gehorsam war hingegen für die erfolgreiche Kriegsführung unabdingbar. ${ }^{30}$ Mit der Härte in der Bestrafung konnte der Orden erreichen, dass insbesondere im dreizehnten im und frühen vierzehnten Jahrhundert eine Disziplin erzielt wurde, die ihresgleichen im ganzen mittelalterlichen Europa suchte.

\section{Korrespondenz:}

Piotr Gotowko,

Anwandstrasse 72, 8004 Zürich, Schweiz piotrg03@yahoo.de

\footnotetext{
28 Perlbach, Statuten 85f., Gesetz 38; GÓRSKI, Studia i szkice 105; SARNOWSKY, Statuten 261; JÓźWIAK, TRUPINA, Organizacja życia 46.

${ }^{29}$ Dies erklärt Schmidt mit der Auslegung des Gesetzes Dietrichs von Altenburg, wo festgehalten wurde, dass man einem Bruder, von dem man wusste, dass er in einen Orden strengerer Observanz übertreten wollte, keine Hindernisse machen durfte; SCHMIDT, Handhabung der Strafgewalt 103f.

${ }^{30}$ GÓRSKI, Studia i szkice 106.
} 


\section{Literatur:}

Hartmut BoocKMANN, Der Deutsche Orden. Zwölf Kapitel aus seiner Geschichte (München 1981).

Marian BISKUP, Gerard LABUDA, Dzieje Zakonu Krzyżackiego w Prusach. Gospodarka Społeczeństwo - Państwo - Ideologia (Gdańsk 1986).

Danielle BuschINGER, Mathieu OLIVIER, Les chevaliers teutoniques (Paris 2007).

Henri DE CURZON, La Règle du temple (Paris 1881).

Alain DemURGER, Die Ritter des Herrn. Geschichte der geistlichen Ritterorden (München 2003).

H. KRAUSE, Die Gesetzgebung, in: HRG ${ }^{1}$, Bd. 1 (Berlin 1971) 1606-1620.

Karol GóRSKI, Zakon Krzyżacki a powstanie państwa pruskiego (Wrocław 1977).

Karol GóRSKI, Studia i materiały z dziejów duchowości (Warszawa 1980).

Karol GóRSKI, Studia i szkice z dziejów państwa krzyżackiego (Olsztyn 1986).

Sławomir JÓźWIAK, Centralne organy władzy, in: Marian BISKUP, Roman CZAJA, Państwo zakonu krzyżackiego w Prusach (Warszawa 2008), S. 123142.

Sławomir JÓźWIAK, Janusz TRUPINDA, Organizacja życia na zamku krzyżackim w Malborku w czasach wielkich mistrzów 1309-1457 (Malbork 2007).

Arno Mentzel-Reuters, Arma spiritualia. Bibliotheken, Bücher und Bildung im Deutschen Orden (Wiesbaden 2003).
Klaus MiLitzer, Von Akkon zur Marienburg. Verfassung, Verwaltung und Sozialstruktur des Deutschen Ordens 1190-1309 (Marburg 1999).

Max Perlbach, Die Statuten des Deutschen Ordens nach den ältesten Handschriften (Halle 1890, ND Hildesheim-New York 1975).

Edward POTKOwSKI, Duchowość Krzyżaków a lektura. Księgozbiory Zakonu Krzyżackiego w Prusach (Warschau 1993).

Jürgen SARNOWSKY, Der Deutsche Orden (München 2007).

Jürgen SARNOWSKY, Die Statuten der geistlichen Ritterorden, in: Gisela DrossBACH, Von der Ordnung zur Norm. Statuten in Mittelalter und Früher Neuzeit (Paderborn München Wien Zürich 2010), S. 181-197.

Gerhard SCHMIDT, Die Handhabung der Strafgewalt gegen Angehörige des Deutschen Ritterordens (Göttingen 1954).

Indrikis STERNS, The statutes of the Teutonic Knights. A study of religious chivalry (phil. Diss., University of Pennsylvania 1969).

Ernst STREHLKE, Tabulae Ordinis Theutonici (Berlin 1869).

Judith UPTON-WARD, The Rule of the Templars (Woodbridge Suffolk 1992).

Johannes VOIGT, Geschichte Preußens von den ältesten Zeiten bis zum Untergange der Herrschaft des Deutschen Ordens, Bd. IX (Königsberg 18271839). 